\title{
¿Qué debemos saber sobre Ciencia?: un ciclo de mejora en la formación científica de los futuros docentes de Primaria
}

\section{What should we know about Science?: a cycle of improvement in the scientific training of preservice Primary teachers}

LIDIA LÓPEZ LOZANO

ORCID: https://orcid.org/0000-0002-5181-638X

Centro adscrito a la Universidad de Sevilla,

Escuela Universitaria de Osuna.

Departamento de Didáctica de las

Ciencias Experimentales y Sociales

lidialopez@us.es

Fecha de recepción: 1-11-2019

Fecha de aceptación:11-11-2019

DOI: http://dx.doi.org/10.12795/9788447221912.058

Pp.: 1328-1348 


\section{Resumen}

Este trabajo presenta un ciclo de mejora en la formación científica de estudiantes de Educación Primaria en la asignatura de Didáctica de las Ciencias Experimentales del 2o curso. Este ciclo abarca parte del primer bloque de contenido de la asignatura dedicado al aprendizaje de la Naturaleza de la Ciencia. El modelo didáctico personal seguido se basa en la Investigación Escolar que implica planificar la enseñanza desde una perspectiva investigativa, crítica y reflexiva e intentando la integración del eje teoría-práctica. Se plantea una secuencia metodológica que parte de la consideración de las ideas del alumnado para el diseño de actividades que propicie un conflicto con éstas y que conduzca al alumnado a nuevos planteamientos. Para ello, se proponen casos y experiencias reales, relevantes que le den sentido a construir un conocimiento fundamentado y les permita desarrollar habilidades y actitudes científicas.

Palabras clave: didáctica de las ciencias experimentales, Grado de Educación Primaria, docencia universitaria, experimentación docente universitaria, alfabetización científica

\section{Abstract}

This work presents a cycle of improvement in the scientific training of Preservice Primary teachers in the subject of Didactic of Experimental Sciences of the 2nd year. This cycle covers part of the first content block of the subject dedicated to learning the Nature of Science. The personal didactic model followed is Inquiry based and involves planning teaching from an inquisitive, critical and reflective perspective trying to integrate the theory-practice axis. A methodological sequence is proposed that starts taking into consideration students' ideas to design activities meant to create a conflict with such ideas leading students to new approaches. We propose real and relevant cases that give meaning and build a grounded knowledge for the development of the students' scientific skills and attitudes.

Keywords: Didactic of Experimental Science, Primary Education Degree, University Teaching, University Teaching Innovation, Scientific literacy. 


\section{Contexto de la intervención}

El Ciclo de Mejora en el Aula (CIMA, en adelante) se propone para la asignatura anual de carácter obligatorio denominada Didáctica de las Ciencias Experimentales (9 ects.) del 2o curso del Grado de Educación Primaria en la Escuela Universitaria de Osuna (centro adscrito a la Universidad de Sevilla). En cuanto a la distribución horaria, la asignatura tiene asignado semanalmente, en horario de mañana, una sesión de dos horas teóricas para el grupo completo y dos sesiones de práctica de una hora cada una, en las que el grupo queda dividido. Sin embargo, durante el desarrollo de la asignatura no se suele distinguir entre sesiones teóricas y prácticas en cuanto al contenido que se aborda, sino que, fundamentalmente, se tiene en cuenta a la hora de organizar las sesiones el aspecto de la división grupal. De esta manera, en las sesiones de grupo reducido, se busca proporcionar al alumnado el espacio para trabajar en aula en detrimento de las tareas no presenciales, contando para su realización con la guía de la docente. El CIMA abarca 10h, 8 sesiones en total.

Respecto al grupo clase, suele caracterizarse por presentar poca formación en ciencias, lo que puede parecer un primer obstáculo en la formación como docentes de ciencias, ya sea por inseguridad con su dominio del contenido (algunos no cursan asignaturas de ciencias desde que dejan de ser obligatorias en la Secundaria) o por rechazo porque la consideran dificil o no les gusta. Esto constituye un importante punto de partida que considerar en nuestra planificación docente. Otro aspecto que se pretende propiciar es el trabajo en equipo, simulando los entornos de trabajo propios de su futura profesión y de la ciencia en sí, ámbito que nos ocupa. Por ello, los estudiantes se organizan por equipos de unos 4-5 componentes. La configuración de estos equipos es responsabilidad de los estudiantes, conformación libre, no obstante, se dan algunas pautas a considerar que los lleven a conformar grupos 
de trabajo viables (criterios de afinidad, cercanía o posibilidad de trabajar o comunicarse fuere del aula, compartir objetivos académicos, etc.).

\section{Diseño previo del CIMA}

En cuanto a la práctica formadora habitual, se mantienen principios adquiridos y/o reforzados durante el curso general de docencia universitaria previo a éste y, en línea con los principios didácticos para el desarrollo de la actividad docente del Departamento de Didáctica de Ciencias Experimentales y Sociales, que actúan como referentes. De tal modo, que la práctica se fundamenta en el principio de isomorfismo (Martín del Pozo, 1994) que no es más (y nada menos) que usar en el aula las mismas estrategias metodológicas que se pretenden enseñar, para que sean puestas en práctica por los aprendices de maestros, cuando ellos tengan su propia aula de Educación Primaria, es decir, una coherencia entre el modelo formativo y el modelo didáctico que se propone para enseñar ciencias. También, se fundamenta en la investigación escolar (Porlán et al., 2010). Si durante la formación pretendemos la aproximación de los futuros maestros y maestras a un Modelo Didáctico de Investigación Escolar (García, 2000) nuestra práctica formativa debe favorecer el aprendizaje basado en la investigación. Y ello, teniendo en cuenta las creencias, ideas y experiencias vividas por los estudiantes fruto de su vida escolar y del plano personal. En este caso, en un Grado de Educación, cobra especial relevancia puesto que los referentes profesionales suelen ser sus profesores/as y sus prácticas docentes vividas durante su etapa escolar. En el diseño de los bloques, se intenta seguir la secuencia: análisis de ideas iniciales - contraste - reflexión/conclusión- reformulación (Abell, Appelton y Hanuscin, 2010; Rivero, Martín del Pozo, Solís y Porlán, 2017). Se prioriza ofrecer el espacio y el tiempo en el aula para desarrollar las actividades, fomentar el debate y la reflexión. Esto 
permite un feedback entre el docente y el alumnado para llevar a cabo una evaluación de carácter formativo.

En líneas generales, se presenta como un reto conseguir un aprendizaje cooperativo, que no agrupados. Otra dificultad es el planteamiento de adecuadas actividades incentivadoras y complejas que les ayude a reformular e identificar debilidades y fortalezas de su propio aprendizaje. En el modelo didáctico deseado está conseguir un tándem metodología-evaluación cuyo sentido sea el de regular y mejorar el proceso de enseñanza-aprendizaje.

\section{Mapa de contenidos}

Para situar el contenido que se pretende enseñar en este CIMA resumimos la secuencia del proceso formativo seguido en la asignatura. Ésta gira en torno a dos grande bloques, el primer bloque (BI) dedicado a la materia objeto de enseñanza: la ciencia; y, el segundo (BII), a la investigación de los problema curriculares relevantes para la profesión, tales como, Contenido escolar, Tratamiento de las ideas de los alumnos, Metodología y Evaluación en ciencias, respondiendo así a “¿Cómo diseñar la enseñanza?"

Esta intervención encaja con el primer bloque "BI: ¿Qué ciencia enseñar?" Éste a su vez contempla dos secciones. La primera, el Bloque 1.1., centrada en la Naturaleza de la Ciencia y el conocimiento científico: ¿Qué es la ciencia y cómo se caracteriza? El CIMA se desarrolla durante esta sección; y la segunda, Bloque 1.2., dedicada a los problemas socioambientales actuales.

En la Figura 1 se muestra el mapa de contenidos para este bloque de trabajo. Se articula en torno a cuatro problemas clave interrelacionados, por lo que la presentación en la trama es flexible. La selección del contenido conceptual responde a trabajar desde la concepción y visión de la ciencia más arraigada en los futuros maestros y maestras (Rivero y Wamba, 2011), de forma que nuestro objetivo será 
ayudarles a que le den coherencia a la enseñanza de la ciencia y la propia naturaleza de la misma. El objetivo es ayudarles a dar coherencia entre la enseñanza de la ciencia y su propia naturaleza.

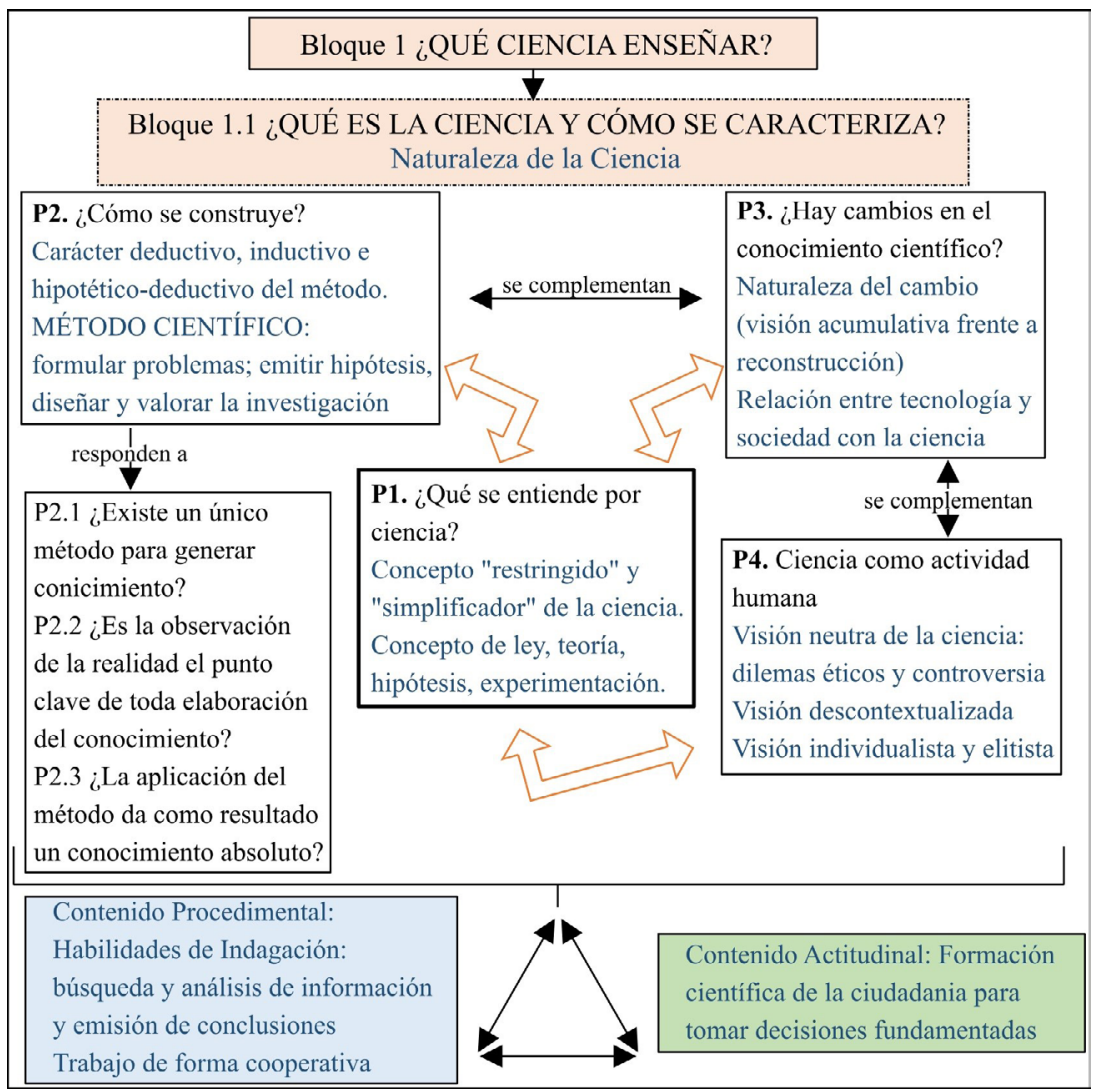

Figura 1. Mapa de contenidos del CIMA

\section{Modelo metodológico y secuencia de actividades}

En la Figura 2 se representa, en el contexto del aula, el engranaje entre la acción de reflexionar sobre las propias ideas de las que se parte en el aprendizaje y la acción de contrastarlas con informaciones nuevas, de manera que, este binomio propicie una evolución en su conocimiento. 


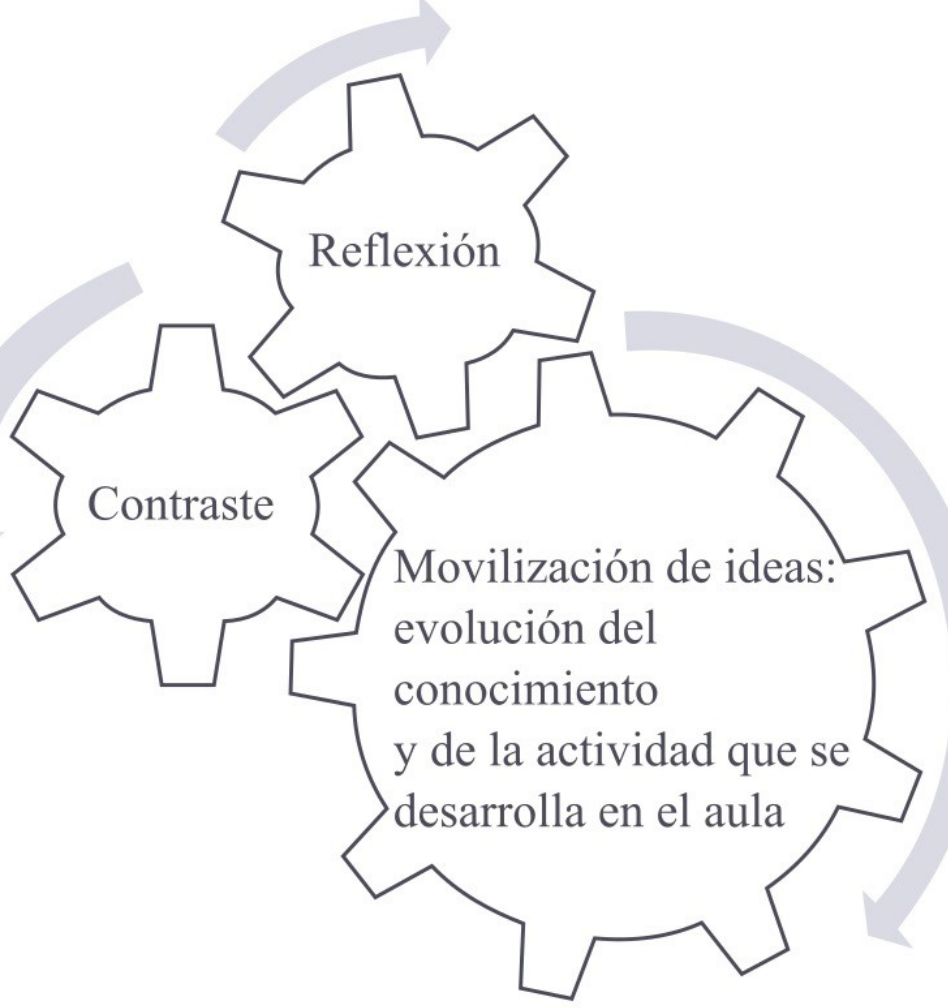

Figura 2. Representación de los pilares metodológicos del CIMA.

Teniendo como referencia lo expuesto en la Figura 2, el modelo metodológico que guía la práctica docente quedaría representado por la secuencia y elementos mostrados en la Figura 3. Las sesiones se plantean desde una perspectiva investigativa, crítica y reflexiva e intentando la integración del eje teoría-práctica. Se caracteriza por plantear inicialmente una problemática relevante para su futura práctica profesional y a partir de ahí se desprende una red de problemas que le den sentido a lo que se va a trabajar. Partimos de sus ideas, intereses y experiencias y se ha diseñado una secuencia de actividades que propicie un conflicto con éstas y que les conduzca a nuevos planteamientos. Para ello las actividades deben proponer casos o experiencias reales, relevantes que le den sentido a 
construir un conocimiento fundamentado y les permita desarrollar habilidades y actitudes. En cuanto a la gestión de aula, se intenta fomentar un clima de clase relajado, basado en el diálogo y la participación activa del alumnado, donde el aula sea el principal espacio de trabajo (se intenta reducir el trabajo no presencial) y donde tenga lugar la explicitación de sus ideas y el desarrollo de la experimentación correspondiente.

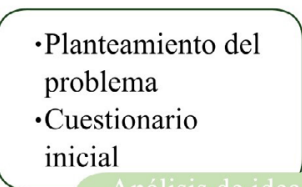

inicial
Contraste de

información

- Aporte de nueva información.

Desarrollo de actividades/

Resolución de casos reales, relevantes y motivadores
Reformulación de las ideas

-Recapitulación y comunicación de lo aprendido: expositivo o escrito.

- Cuestionario final

Figura 3. Modelo metodológico del CIMA presentado.

La secuencia de actividades está programada para favorecer el cuestionamiento de las concepciones mayoritariamente arraigadas en los estudiantes sobre la ciencia (Rivero y Wamba, 2011) y poder construir una imagen válida de ésta a lo largo de la intervención. La secuencia diseñada para el CIMA se recoge resumidamente en la Tabla 1. Se ha señalado en cursiva el problema clave que se trabaja principalmente, aunque por la propia naturaleza del contenido se abordan conjuntamente todos los problemas planteados. También, mediante un código de color se identifica el tipo de actividad según lo expresado en la Figura 3. Se proponen actividades tanto de tipo individual como de equipo. 


\section{Tabla 1. Secuenciación de actividades del CIMA}

S1 Se inicia presentando el bloque I y cumplimentando el Cuestionario de ideas

(1h) iniciales (escalera de aprendizaje)

Reflexión inicial y puesta en común de sus ideas iniciales.

Introducción del contenido Lectura de varios textos de Rivero y Wamba (2011).

Se trabaja en gran grupo diversos textos que abordan los cuatro problemas

planteados. Funciona como base para abordar la temática, empezar

contrastando sus visiones con informaciones nuevas y rigurosas. Puesta en

S2 común. Permite trabajar la caracterización de la Naturaleza de la Ciencia

(2h) (Preguntas claves): P1, P2, P3 y P4.

De manera individual, se retoma una de las cuestiones que se mantendrá

abierta para su reformulación a lo largo del bloque 1.1 en la que se debe

argumentar cómo se construye el conocimiento científico: Act. Reformulación

de la representación del proceso de elaboración del conocimiento. P2. ¿Cómo

se construye?

Caso práctico: Noticias/anuncios recientes con temáticas científicas.

De una actividad de lectura comprensiva pasamos a una más visual y práctica en el contexto más próximo. Como introducción a la actividad de indagación que deben realizar se les presentan dos casos en el contexto de la publicidad en los que el uso de la ciencia está cuestionado. Se lleva a cabo mediante exposición dialogada y apoyada en medio audiovisual.

S3 Analizamos diferentes criterios: neutralidad, fuentes, estética, tendencias, etc.

(1h) Para este "desmontaje" y/o caracterización, se usa un dossier con preguntas para reflexionar sobre ambos casos. Esta tarea conduce a la propuesta de actividad de indagación. Se les proponen tres productos como casos de estudio para averiguar si se trata o no de marketing pseudocientífico. Pretendemos trabajar principalmente el P4 (desarrollo de habilidades de indagación científica y de formación científica, actitudes sobre y en ciencia) e insistir en la formación científica como medio para fomentar una ciudadanía con cierto criterio crítico ante los asuntos del entorno.

Resolución del caso práctico. La clase se agrupará en tres equipos, se les proporciona un guion con los pasos a seguir para nuestra investigación y deberán alcanzar unas conclusiones sobre la idoneidad del producto.

- Guion-Actv. "Des-montado por la Ciencia": Marketing pseudocientífico (López-Nicolás, 2016).

S4 Estas conclusiones nos llevan a tres opciones: engaño ilegal, legal pero poco

(2h) o nada ético o legal y veraz (López Nicolás, 2016). Se abordan los dilemas éticos en la ciencia (al hilo de las lecturas iniciales). Además, del conceptual, se trabaja principalmente contenido del tipo procedimental y actitudinal. Deben hacer búsquedas de los componentes y búsquedas bibliográficas en repositorios científicos (dificultad baja). Es una actividad que a su vez sirve de experiencia experimental, permite trabajar la construcción del conocimiento científico $(P 2)$

Jornadas de Formación e Innovación Docente del Profesorado | № 2 (2019) Esta obra se distribuye con la licencia Creative Commons Reconocimiento-NoComercial-SinObraDerivada 
Llegado a este punto, se recopilan las ideas tratadas. La docente guía

la recopilación en base a las respuestas de los estudiantes (exposición

S5 dialogada)

(1h) Seguidamente, presentación de nueva actividad: Lectura de textos sobre las investigaciones médicas reales de dos científicos (Nieda, 2012) y planteamiento de cuestiones sobre el texto. Trabajo individual.

Resolución de las cuestiones planteadas de la lectura. Debate grupal sobre

S6 los aspectos que caracteriza la actividad científica, se trabaja tanto las fases

(2h) del método científico como los factores que influyen en el devenir de una

investigación científica. P2, P3 y P4; formación científica (actitudes sobre y en ciencia)

Actividad experimental sencilla en el aula sobre una investigación (balanza). Guion-Act. Diseño de una investigación: planteamiento del problema, emisión

S7 de hipótesis, diseño experimental y valoración y conclusiones.

(1h) Trabajo por equipos.

P2. ¿cómo se construye el conocimiento científico?; desarrollo de habilidades de indagación científica

S8 Cuestionario final (escalera de aprendizaje) con el objetivo de conocer si ha

(1h) habido movilización de sus ideas y concepciones y en qué grado.

\section{Valoración del proceso: cuestionario inicial y final}

Al tratarse de un ciclo de mejora de breve duración y, sobre todo, por abarcar parcialmente un bloque, se contempla una evaluación fundamentalmente de carácter cualitativo. Al hilo de lo expuesto, el instrumento principal para la valoración del aprendizaje del alumnado será el cuestionario inicial y final que permitirá conocer las posibles evoluciones del conocimiento de los estudiantes sobre los aspectos más relevantes tratado mediante las escaleras de aprendizaje (Porlán, 2017). Por ello, se exponen en la Tabla 2 las cuestiones que conforman dicho cuestionario junto al problema (Figura 1) con el que se vincula. Este tratamiento permitiría a su vez, detectar las posibles dificultades de aprendizaje, lo que es tremendamente útil para poder reconducir el proceso formativo antes de acabar el bloque. Y, además, poder inferir en el grado de efectividad de las actividades propuestas al final del proceso

Jornadas de Formación e Innovación Docente del Profesorado | № 2 (2019) Esta obra se distribuye con la licencia Creative Commons Reconocimiento-NoComercial-SinObraDerivada Internacional (CC BY-NC-ND 4.0.) 
de enseñanza-aprendizaje. Ello se enmarcaría en una evaluación de tipo formadora.

Tabla 2. Cuestionario inicial y final y su vinculación con los problemas claves.

\begin{tabular}{|l|l|}
\hline Cuestiones que se proponen & Problema \\
\hline 1. ¿Qué entiendes por ciencia? & P1 \\
\cline { 2 - 3 } 2. ¿Cómo crees que es el proceso mediante el cual los científicos & P2 \\
\cline { 2 - 2 } elaboran conocimiento? Represéntalo mediante un esquema y & P2, P3, P4 \\
explícalo brevemente. & \\
3. Comenta las siguientes afirmaciones, expresando razonada y & \\
detalladamente su grado de veracidad: \\
a) El papel más importante en el proceso de elaboración del \\
conocimiento científico lo tienen la observación rigurosa de los \\
fenómenos de la naturaleza. \\
b) Todos los conocimientos científicos son comprobables \\
directamente en la realidad. \\
c) La actividad científica es una actividad neutra, no \\
"contaminada" por intereses personales o sociales. \\
d) El conocimiento científico es superior a otros tipos de \\
conocimientos pues se trata de un conocimiento verdadero. \\
e) El conocimiento científico actual es esencialmente igual al que \\
existía hace 100 años, sumándole todos aquellos conocimientos \\
que se han elaborado desde entonces hasta ahora. \\
\end{tabular}

Fuente: Adaptado de Rivero, Martín del Pozo, Solís y Porlán (2017). Nota: P1, P2, P3, P4 son los problemas planteado en el mapa de contenidos en la Figura 1.

Estos resultados de aprendizaje obtenidos del cuestionario se completarán con las evidencias resultantes de la resolución de las actividades propuestas durante el ciclo de mejora que quedarían recogidas en un porfolio. El propósito es relacionar la evolución o cambio que se desprenda del cuestionario con la resolución de las actividades teniendo como referencia el problema que se trata en éstas. De modo que, ayude a inferir el grado de coherencia entre lo declarado y lo propuesto. 


\section{Aplicación del CIMA}

El inicio del CIMA coincide con el inicio de la asignatura y del curso. Esto condujo a una tímida predisposición a participar en las primeras puestas en común. Cumplimentaron el cuestionario inicial con cierta preocupación por la idoneidad de sus respuestas. Ante esto, para darles mayor seguridad, la docente aclaró la necesidad de ser conscientes de lo que uno piensa (y no tanto lo que recuerda académicamente) sobre las cuestiones que va a trabajar, como su punto de partida en el aprendizaje. También, algunos mostraron cierta dificultad para comprender lo que se requería. Se aclararon las dudas.

En la siguiente sesión, mediante una puesta en común, iniciamos el bloque de trabajo poniendo en pie sus respuestas acerca de lo que entienden por ciencia y cómo la caracterizan. Esto da paso a trabajar con extractos de textos históricos como contraste a las creencias mayoritarias. Para su lectura en clase éstos se reparten entre el estudiantado (aunque disponen del texto completo en Plataforma). La docente conduce la actividad exponiendo interrogantes-situaciones (p.e. ¿cuántas observaciones podemos considerar suficientes?) vinculado a un texto histórico que ayuda a desarrollar la idea, que es leído por un estudiante y explica lo entendido. Intervención libre por parte del alumnado. En general, baja participación, cierta dificultad en la lectura y su comprensión. Hubo una alumna que se limitó a leer el texto, sin argumentar. Por otro lado, les llamó positivamente la atención los sucesos.

Para la siguiente actividad solicité que se agruparan en 3 grandes equipos (uno por producto a analizar) resultando 20-21 componentes. En esta sesión se trabaja la ciencia desde la perspectiva social, en el contexto de la publicidad. El objetivo es que cada equipo investigue sobre el uso de la ciencia en la publicidad de un producto alimenticio, para ello primeramente introduzco dos casos, cuyo análisis conduce a la propuesta de indagación grupal, se les presenta el informe y aclaramos los puntos que 
lo configuran y el procedimiento de trabajo en el aula. En este punto, resaltaría que la instrucción se diseñó para que los pasos a seguir en la indagación, descritos en el informe que debían elaborar, saliesen de manera "natural" y gradual colectivamente tras la previa exposición-introducción de dos casos, pero no fue así. Fue preciso explicar qué hacer y cómo hacerlo; incluso cómo cumplimentar cuestiones que se describían en el informe. Resultó más dirigido de lo esperado. El informe de esta actividad es de entrega individual, aunque para resolverlo se necesita del trabajo de indagación en equipo, fundamentalmente para resolver las dos primeras partes (información sobre el producto), la tercera era una reflexión conclusiva final y personal. De manera que se propiciaba que todos estuviesen implicados o activos en la indagación, ya que de eso dependía su informe, también la capacidad de organización y planificación del trabajo. Se dedicó la sesión de $2 \mathrm{~h}$ de clase al trabajo en equipo. Esta actividad, quizás por ser la primera de indagación, presentó dificultad para trabajar de manera autónoma, suelen esperar recibir hasta la más mínima indicación. Dificultad en distinguir los componentes del producto, en saber qué es una referencia, en plasmar lo resultados alcanzados. Operacionalmente dan con datos (siguen los pasos como si fuese una receta), pero no saben integrarlos en el informe, darle significado (me preguntaban "he leído esto y tal..., pero y ¿ahora qué hago?"). Dificultad en alcanzar conclusiones, algunos tendían a buscar en web un artículo o post sobre el producto para poder resolver, aun habiendo dado con respuestas. Sobre la intra-organización y planificación del trabajo, la tendencia inicial era repartirse la elaboración de las diferentes partes del informe en línea a lo que acostumbran, en lugar de repartirse contenido sobre el que indagar (más efectivo por el planteamiento del informe). Se supo reconducir esta situación como parte del aprendizaje. No obstante, en este punto, ya la participación en clase es mayor y se propician discusiones sobre lo que se está tratando y se muestran entusiasmados con el enfoque dado al tema. 
Relacionado con esto y, respecto a la planificación inicial, no dio tiempo a realizar la actividad experimental ya que se alargó la puesta en común de los resultados obtenidos por los equipos y, en consecuencia, la correspondiente a la actividad sobre las investigaciones reales. Recapitulando ambas, se fueron esbozando las implicaciones sociales del uso de la ciencia, responsabilidades, uso de las RRSS como su principal fuente de información, necesidad de adquirir una actitud crítica. Se finalizó con la cumplimentación final del cuestionario.

\section{Evaluación del aprendizaje de los estudiantes}

Para abordar este punto, es necesario aclarar que no se ha podido finalizar el análisis del portfolio por cuestión de tiempo y volumen de informes. Así para poder valorar si ha habido evolución en las ideas del alumnado como consecuencia de la aplicación del CIMA diseñado analizamos las respuestas dadas al cuestionario al inicio y al final. Para ello usamos la representación gráfica de la escalera de aprendizaje (Porlán, 2017) mostrando los datos en ambos momentos para cada cuestión propuesta.

Sobre el concepto que tienen de la ciencia, C1. ¿Qué entiendes por ciencia?/ Categoría: grado de conceptualización de la ciencia.

\begin{tabular}{|c|c|c|c|}
\hline & \multicolumn{2}{|c|}{$\begin{array}{l}\text { No entenderla como actividad } \\
\text { humana en respuesta a los problemas } \\
\text { del mundo }\end{array}$} & \multirow{2}{*}{$\begin{array}{l}\text { F:26.5\% } \\
\text { I:25\% } \\
\text { N3. Conceptualización amplia. } \\
\text { Disciplina o estudio de los fenómenos } \\
\text { del mundo que nos rodea. Obtenemos } \\
\text { explicaciones razonadamente. }\end{array}$} \\
\hline $\begin{array}{l}\text { Reducirla a la } \\
\text { método cientifi } \\
\text { tipos de saber }\end{array}$ & $\begin{array}{l}\text { mera aplicación de } \\
\text { ico sin considerar }\end{array}$ & $\begin{array}{l}\text { F: } 24.5 \% \\
\text { I: } 17.3 \%\end{array}$ & \\
\hline & $\begin{array}{l}\mathrm{F}: 49 \% \\
\mathrm{I}: 40.4 \%\end{array}$ & $\begin{array}{l}\text { N2. Se conceptua } \\
\text { tipos de saberes }\end{array}$ & $\begin{array}{l}\text { liza identificando distintas disciplinas, } \\
\text { sociales, química, física). }\end{array}$ \\
\hline $\begin{array}{l}\mathrm{F}: 0 \% \\
\mathrm{I}: 17.3 \%\end{array}$ & \multicolumn{3}{|c|}{$\begin{array}{l}\text { N1. Conocimientos fruto de la aplicación de los pasos del método científico, } \\
\text { basado en la observación-experimentación, lo describen. }\end{array}$} \\
\hline
\end{tabular}

Figura 4. Escalera de aprendizaje correspondiente a la cuestión 1. 
Tal y como muestra la Figura 4, se da cierta evolución en su conceptualización de la ciencia, aunque básicamente se refuerza la idea de la ciencia como conocimiento basado en los hechos observables y la experimentación (N1) que lleva implícita la idea de método científico. Prevalece la estructura formal de la ciencia frente a una visión más amplia que incluya su dimensión práctica y social (N3).

En relación a cómo se construye, C2. ¿Cómo crees que es el proceso mediante el cual los científicos elaboran conocimiento? Categoría: grado de conocimiento de construcción del conocimiento científico.

\begin{tabular}{|c|c|c|c|}
\hline & \multicolumn{2}{|c|}{$\begin{array}{l}\text { No contemplar la construcción } \\
\text { como un proceso abierto y } \\
\text { complejo }\end{array}$} & $\begin{array}{l}\mathrm{F}: 0 \% \\
\mathrm{I}: 0 \%\end{array}$ \\
\hline \multicolumn{2}{|c|}{$\begin{array}{l}\text { Basarse en la existencia } \\
\text { de un único método } \\
\text { cientifico }\end{array}$} & $\begin{array}{l}\mathrm{F}: 12.3 \% \\
\mathrm{I}: 7.7 \%\end{array}$ & $\begin{array}{l}\text { N3. Como un proceso complejo, } \\
\text { abierto, flexible, mezcla de } \\
\text { lógica y creatividad. }\end{array}$ \\
\hline $\begin{array}{l}\text { F:87.7 } \\
\text { I:84.6\% }\end{array}$ & & \multicolumn{2}{|c|}{$\begin{array}{l}\text { N2. Descripción cualitativa de cómo proceden } \\
\text { (meticulosamente, organizado, partiendo de su interés, } \\
\text { inquietud, basado en anteriores investigaciones). }\end{array}$} \\
\hline $\begin{array}{l}\text { N1. Visión i } \\
\text { y con mayor }\end{array}$ & $\begin{array}{l}\text { ductivista. } \\
\text { menor aci }\end{array}$ & $\begin{array}{l}\text { criben el método } \\
\text { (p.e. confusión }\end{array}$ & $\begin{array}{l}\text { ntífico más o menos desarrollado } \\
\text { re el término hipótesis). }\end{array}$ \\
\hline
\end{tabular}

Figura 5. Escalera de aprendizaje correspondiente a la cuestión 2.

Como anteriormente, se advierten según Figura 5, tímidos cambios en positivo y, en concordancia con la postura mostrada en su visión de la ciencia, se refuerza (y se mejora su descripción) el método científico como explicación del proceso de construcción del conocimiento. Principalmente, al final, incluyen a la comunidad científica como parte del proceso y la importancia de su divulgación como pasos finales. Ninguno lo describe como un proceso abierto en el que tiene cabida la creatividad, el azar u otros factores sociales (N3). Elevan la observación al paso fundamental. También se advierte una potente confusión sobre el significado de hipótesis y su relevancia o rol en el proceso. 

planteaban 5 afirmaciones:

\section{Categoría: grado de inductivismo, la observación como papel principal en una investigación}

Desacuerdo I:21.2\%

Desacuerdo F:38.8\%

N2. Otorgan misma importancia a todos los pasos del proceso /Para unos, el desacuerdo no se debe al papel de

Acuerdo F:61.2\% la observación sino al objeto de estudios -los fenómenos naturales: falso por haber considerado solo fenómenos

Acuerdo I:78.8\% naturales. Además, se da confusión conceptual.

N1. La observación es lo más importante ya No se consideran las limitaciones que conlleva el que así se inicia el proceso, además lo observable es verificable.

proceso de la observación

Figura 6. Escalera de aprendizaje correspondiente a la cuestión 3a.

Categoría: grado de concepción empírica de la ciencia:

Desacuerdo I:38.5\%

Desacuerdo F:29.2\%

N2. No todo puede basarse en la realidad (lo que se ve). No obstante, confusión acerca de lo que se entiende por realidad (laboratorio vs realidad), conocimiento teórico

Acuerdo I:61.5\%

Acuerdo F:70.8\% como algo aleatorio, sin base alguna "real" (se ve), p.e. lo que no se puede ver, como los agujeros negros.

N1. Son comprobables en la realidad porque parte de la observación de la realidad, en linea a su percepción de cómo se construye el conocimiento.

No entender el estudio cientifico basado en modelos como representaciones de la realidad

Figura 7. Escalera de aprendizaje correspondiente a la cuestión 3b.

\section{Categoría: grado de percepción de neutralidad de la ciencia}

\begin{tabular}{|c|c|}
\hline & Desacuerdo I:65.4\% \\
\hline Acuerdo F:18.4\% & $\begin{array}{l}\text { N2. Influyen intereses de otro ámbitos, pero también } \\
\text { porcentaje considerable (sobre todo al inicio) se han } \\
\text { tomado la declaración entendiendo "interés personal } \\
\text { como interesante o motivante para el que investiga. } \\
\text { acuerdan es neutra la contaminan otros ámbitos. lleg } \\
\text { declarar que los científicos falsean sus datos por alca } \\
\text { reputación. La empresa farmacéutica es recurrente c } \\
\text { ejemplo para su postura al inicio. COnfunden uso de } \\
\text { ciencia con la ciencia en si. }\end{array}$ \\
\hline $\begin{array}{l}\text { N1.Es un conocimiento basado en lo } \\
\text { demonstrable, verdadero, por tanto es neutral } \\
\text { y objetiva. }\end{array}$ & $\begin{array}{l}\text { No considerar la ciencia como actividad humana } \\
\text { sujeta a subjetividades y al contexto en el cual se } \\
\text { desarrolla }\end{array}$ \\
\hline
\end{tabular}

Figura 8. Escalera de aprendizaje correspondiente a la cuestión 3c. 
Categoría: grado de percepción como conocimiento verdadero:

\section{Acuerdo I:69.2\%}

Acuerdo F:55.1\%

N1. El desarrollo del méetodo científico así lo hace, es demonstrable por tanto verdadero por tanto superior a lo no demonstrable.
Desacuerdo I:30.8\%

Desacuerdo F:44.9\%

N2. Es verdadero en su ámbito pero entran en juego los "otros" conocimientos (citan el religioso) mientras sea "verdadero" ya es equiparable a otro conocimiento. Se cuestiona la superioridad por el hecho de ser verdadero.

Derivado de la primacía de la observación: "ver para creer". Lo consideran cierto y definitivo.

Figura 9. Escalera de aprendizaje correspondiente a la cuestión 3d.

Categoría: grado de consideración evolutiva de la ciencia:

\begin{tabular}{|c|c|}
\hline & Desacuerdo F:76.6\% \\
\hline Acuerdo F:23.4\% & $\begin{array}{l}\text { N2. Mayoritariamente se identifica con los avances en la } \\
\text { tecnología e instrumentación. al final se alude a la } \\
\text { evolución en el estudio. en la sociedad. }\end{array}$ \\
\hline $\begin{array}{l}\text { N1. El conocimiento es el mismo, lo que se } \\
\text { "demonstró" es verdadero e inutable, lo que } \\
\text { cambia son las tecnologías. }\end{array}$ & $\begin{array}{l}\text { Considerar el conocimiento cientifico como un } \\
\text { producto cerrado y acabado. }\end{array}$ \\
\hline
\end{tabular}

Figura 10. Escalera de aprendizaje correspondiente a la cuestión 3e.

Las escaleras muestran evolución disminuyendo los acuerdos (excepto una) y aumentando los desacuerdos tras la intervención, aunque no ha habido sorpasso entre ambos momentos. Así, los resultados se prestan a una valoración global que caracterice la visión de nuestro alumnado. Las posturas mostradas en las dos primeras cuestiones suelen ir en concordancia y ligadas a su idea de "método científico" como obtención del conocimiento científico junto a la cuestión $3 d$ (ciencia como conocimiento verdadero). La visión de los estudiantes se enmarca en las más arraigadas sobre la naturaleza de la ciencia según Rivero y Wamba (2011), nivel inicial 1, si bien aumenta la discrepancia al final, además, se advierte un leve cuestionamiento acerca de la ciencia como conocimiento verdadero aludiendo al papel de la comunidad científica y manejando el término "validez". La discrepancia aumenta 
sobre la falta de neutralidad y de cambio. Sobre éstas, parecen más evidente para ellos por los avances tecnológicos experimentados, no obstante, la visión de cambio es fundamentalmente acumulativa. Y, por los conflictos de intereses en las industrias, aunque también suelen entender la dependencia del interés personal o social tomándola como actividad "interesante" y motivadora para el científico, reinterpretando la declaración.

Se aprecia la incorporación a su argumentación de algunos de los casos trabajados, aunque no siempre acertadamente, para justificar su respuesta. Sobre las argumentaciones, en general, son débiles e incluso confusas, sobre todo al inicio, usando términos inadecuadamente o mostrando falta de comprensión lectora. Observando las figuras, hay ciertas creencias más fáciles de superar que otras -en la ciencia influyen diversos factores ambientales, políticos, ideológicos, sociales, etc...y se trata de un conocimiento cambiante-. Sobre el resto, concuerda con lo obtenido en las dos cuestiones anteriores y están fuertemente asumidas. Por tanto, habría que reforzar las actividades deteniéndonos concienzudamente en los conceptos más básicos, casi partiendo de su significado (observación, hipótesis, teoría, ley, realidad, experimentación, objetividad, conocimiento).

\section{Evaluación del CIMA puesto en práctica}

Acerca del aprendizaje del alumnado, a la luz del análisis de sus respuestas parece que se inicia con una idea o recuerdo vago sobre ciencia y el método científico como contenido central de la temática (al inicio apenas se nombra como tal, pero es implícito en sus descripciones) y, al final, parece que se encuentran en ese punto de poner en pie eso que les "sonaba o recordaban" de las clases de ciencia. A juzgar por las escaleras, la escasa movilidad de niveles nos lleva a concluir que el cambio conceptual es 
lento y los obstáculos potentes. Durante la intervención inicial van dándole forma a sus creencias de partida según interpretan lo que han leído y trabajado en las sesiones. Considero que para poder superar esta visión simple y reduccionista de la ciencia, primero deben entender las ideas más básicas, lo que dificulta avanzar en el grado de conocimiento.

El final del CIMA se podría considerar como un momento intermedio en el proceso de aprendizaje - de hecho, es la primera mitad del bloque- convendría volver a plantear estas cuestiones finalizado el bloque completo, en el que se experimenta un proyecto de indagación y se aborda la ciencia vinculada a las problemáticas socioambientales enlazando su vinculación con la educación científica. Sostengo que ayudaría a ampliar la conceptualización de ciencia en su dimensión práctica y social. Por lo que se presenta como un buen inicio del proceso enseñanza-aprendizaje de este contenido viendo la gradualidad del proceso de cambio.

Por ello, contemplo algunas modificaciones futuras. En cuanto a las actividades planteadas, considero necesario mejorar la primera dedicada a los textos sacados de la historia de la ciencia incluyendo un tratamiento didáctico más amplio ya que no resulta eficaz hacerlo solo mediante puesta en común grupal leyendo los fragmentos históricos. Es necesario trabajar sobre la aclaración de ciertos términos (hipótesis, teoría, tesis, experimentación, realidad...) dada su confusión. Tal vez el replanteamiento inicie por hacer más explícito los interrogantes que se podrían responder, como hilo conductor de la actividad (Bain, 2007). Por otro lado, no ha sido posible la reelaboración formal de la cuestión P2, sí de manera implícita conforme se avanzaba en el tema.

Sobre el cuestionario, incluir en la versión final cuestiones relativas a su propia percepción de cambio en el 
aprendizaje y sobre qué aspectos, además de preguntar por aquella actividad que considere que le ha ayudado a aclarar conceptos. En cuanto a la metodología de cumplimentación, también sería necesario cambiar la vía, usar el tradicional papel o usando formulario online, ya que la entrega de documento por Plataforma Virtual se ha prestado a que algunos mandasen el mismo documento que el inicial.

Por último, señalar que los principios didácticos que han guiado toda la experiencia se muestran adecuados no solo para dinamizar las sesiones de clase, crear un ambiente de trabajo distendido y basado en la confianza y dar, así, mayor protagonismo al estudiante en su proceso de aprendizaje, sino también como precursor de éste. Hay

que tener en cuenta la poca familiaridad que los estudiantes tienen con esta metodología -partir de sus ideas, tomar conciencia de ellas y trabajar de manera autónoma para reformularlas- cuyo momento de aplicación en este CIMA, además, coincide con el inicio de la asignatura, lo que implica un cambio más lento que puede traducirse en resultados menos ambiciosos y requiere, a su vez, mayor apuesta por su aplicación.

Jornadas de Formación e Innovación Docente del Profesorado | № 2 (2019) Esta obra se distribuye con la licencia Creative Commons Reconocimiento-NoComercial-SinObraDerivada 


\section{Referencias bibliográficas}

Abell, S. K., Appleton, K., y Hanuscin, D. (2010). Designing and teaching the elementary science methods course. New York: Teaching and Learning in Science Series.

Bain, K. (2007). Lo que hacen los mejores profesores universitarios. Valencia: Servicio de Publicaciones de la Universidad de Valencia

García Pérez, F. F. (2000). Los modelos didácticos como instrumento de análisis y de intervención en la realidad educativa. Biblio 3W. Revista Bibliográfica de Geografía y Ciencias Sociales, 207. En: http://www.ub.edu/geocrit/b3w-207.htm.

López Nicolás, J.M. (2016). Vamos a comprar mentiras. Alimentos y cosméticos desmontados por la Ciencia. Palencia: Cálamo

Martín del Pozo, R. (1994). El conocimiento del cambio químico en la formación inicial del profesorado. Estudio de las concepciones disciplinares y didácticas de los estudiantes de Magisterio. Tesis doctoral. Universidad de Sevilla.

Nieda, J. (2012). Jenner y Semmelweis, dos genios con diferente fortuna. Revista Alambique Didáctica de las Ciencias Experimentales, 72, 28-36.

Porlán, R. (coord.) (2017). Enseñanza universitaria. Cómo mejorarla. Madrid: Editorial Morata.

Porlán, R., Martín del Pozo, R., Rivero, A., Harres, J., Azcarate, P., y Pizzato, M. (2010). El cambio del profesorado de ciencias I: Marco teórico y formativo. Enseñanza de Las Ciencias, 28(1), 31-46.

Rivero, A., Martín del Pozo, R., Solís, E. y Porlán, R. (2017). Didáctica de las ciencias experimentales en educación primaria. Madrid: Sintesis.

Rivero, A., y Wamba, A.M. (2011). Naturaleza de la ciencia y construcción del conocimiento científico. Biología y Geología. Complementos de formación disciplinar (pp. 9-26). 